\title{
The ATLAS Hadronic Tau Trigger: Initial Run-2 Strategy and Performance
}

\author{
Mark Pickering ${ }^{1}$ \\ On behalf of the ATLAS Collaboration \\ University of Oxford, United Kingdom \\ E-mail: mark.andrew.pickering@cern.ch
}

As proton-proton collisions at the LHC reach instantaneous luminosities of over $10^{34} \mathrm{~cm}^{-2} \mathrm{~s}^{-1}$, and a centre-of-mass energy of $13 \mathrm{TeV}$, the strategies for triggering have become more important than ever for physics analyses. In these conditions, single tau lepton triggers suffer from severe rate limitations, despite the sophisticated algorithms used in the tau identification. The development of further fast algorithms and the design of topological selections are the main challenges to allow a large program of physics analysis. The tau triggers provide many opportunities to study new physics beyond the Standard Model, and to get precise measurements of the properties of the Higgs boson decaying to tau leptons. Presented here is the strategy for Run-2 data-taking and the initial performance of the ATLAS tau trigger with a dataset corresponding to an integrated luminosity of $78.3 \mathrm{pb}^{-1}$ at $\sqrt{\mathrm{s}}_{\mathrm{s}}=13 \mathrm{TeV}$.

XXVII International Symposium on Lepton Photon Interactions at High Energies 17-22 August 2015

Ljubljana, Slovenia

${ }^{1}$ Speaker 


\section{Introduction}

Tau leptons are a signature of many Standard Model and beyond the Standard Model searches of the ATLAS experiment [1], including the decay of the Higgs boson. Tau leptons decay either hadronically, around $65 \%$ of the time, or leptonically, with their relatively short lifetimes resulting in a decay inside the beam pipe. The tau trigger aims to detect hadronic tau decays, since tau leptons decaying to electrons or muons are detected by the respective electron and muon triggers. The short lifetime and difficulty in distinguishing tau jets from quark or gluon initiated jets makes the reconstruction, identification and triggering of hadronically decaying tau leptons a challenge. Characteristic features of hadronic tau decays are one or three charged pion tracks in a collimated jet coming from a displaced vertex.

The Run-2 ATLAS trigger system is designed to reduce a $40 \mathrm{MHz}$ rate of proton-proton collisions in the LHC to a rate of $1 \mathrm{kHz}$ for events being written out to data storage. The increased centre-of-mass energy and instantaneous luminosity of Run-2 pose many challenges for the identification of hadronic tau decays within time and bandwidth constraints. As such, the tau trigger strategy has undergone significant changes compared to Run-1.

\section{ATLAS Run-2 Trigger}

In Run-2, ATLAS uses a two tier trigger system [2]. The first tier, level 1 (L1), is hardware based. Regions of interest (RoIs) are identified using information from the muon spectrometer and the calorimeters. These RoIs are passed on to the software based High-LevelTrigger (HLT) at a rate of up to $100 \mathrm{kHz}$. Here, object reconstruction takes place using algorithms similar to those used in final data processing.

The ATLAS trigger system in Run-2 has undergone a series of upgrades during the recent long shutdown [3]. Bandwidths have increased at both L1 and HLT. The L1 tier has improved significantly, with a new central trigger processor enabling a greater number of trigger combinations and thresholds, and a new topological trigger module enabling topological selections between L1 objects. The level 2 and event filter tiers of the Run-1 trigger have been merged into a single HLT tier, enabling resource-sharing and more complicated reconstruction algorithms to take place.

\section{Tau Trigger Strategy in Run-2}

The tau trigger has undergone various changes in strategy in order to cope with increased rates during Run-2. RoIs are identified by first applying cuts on the transverse energy deposits in summed electromagnetic and hadronic calorimeter towers defined at L1, with the option of a variable cut on the transverse energy in an "isolation" ring of calorimeter towers around this core. It is also possible to apply topological cuts, such as angular separations or overlap removal, between L1 tau objects and other L1 objects such as electrons, missing transverse energy or other taus. The RoIs identified in the calorimeter are then passed on to the HLT where the position and transverse energy deposition are refined, and a cut is placed on the deposited energy in the "Topo-Clustering" stage [4]. A fast computation of track parameters 
similar to the level 2 tracking in the Run-1 trigger occurs, and a "FastTracking" algorithm counts and cuts on track multiplicities, requiring one to three charged tracks in a core region, and no more than one track in an isolation region around the tau candidate. Only tracks with a transverse momentum greater than $1 \mathrm{GeV}$ are considered. Finally full HLT track reconstruction is carried out and along with shower shape variables fed into an online (trigger level) Boosted Decision Tree (BDT) algorithm similar to that applied in final reconstruction. This algorithm selects taus according to three levels of purity, 'loose', 'medium' and 'tight'.

An overall improvement in the efficiency of the tau trigger to identify tau leptons with respect to final event reconstruction is expected in Run-2. This results from the new high-leveltrigger strategy which includes a more precise energy calibration, a faster tracking stage and an online tau identification algorithm closer to the one used in offline tau reconstruction.

\section{Performance of the ATLAS Tau Trigger in Early Run-2 Data}

\subsection{Level 1 Trigger Rates Versus Luminosity}

Figure 1, shows the rates of the level 1 single and combined tau triggers as a function of instantaneous luminosity measured in ATLAS. In order to reduce the rates at L1, tau objects are combined with other L1 objects. 'TAU', 'EM', 'J' and 'XE' indicate the type of L1 object: tau, electron, jet or missing energy respectively. The digit before these names indicates the object multiplicity, while the digits after correspond to the transverse energy requirement, e.g. L1_TAU20_2TAU12 contains at least two taus with a transverse energy of greater than $12 \mathrm{GeV}$, at least one of which also has a transverse energy greater than $20 \mathrm{GeV}$. 'IM' refers to the isolation level in the isolation ring around the tau candidate, and ' $\mathrm{HI}$ ' indicate that a hadronic isolation cut is applied. The data were collected at a center-of-mass energy of $13 \mathrm{TeV}$, from the 6 th to the 12 th of July 2015 , with a spacing of 50 ns between proton bunches.

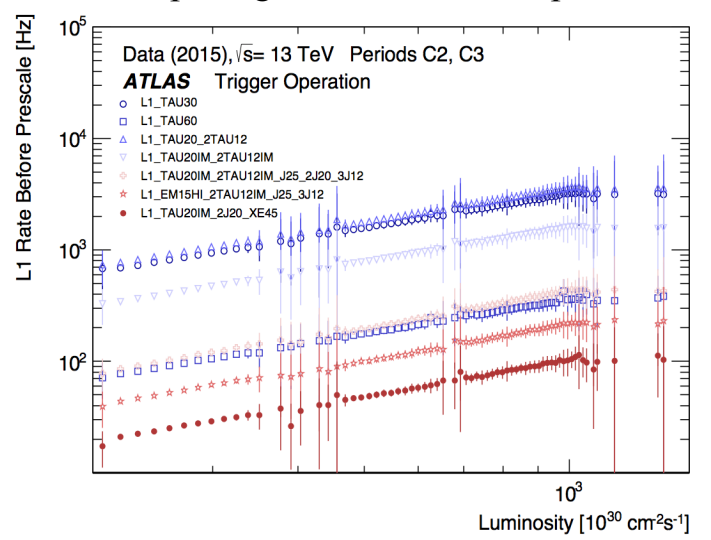

Figure 1: ATLAS Run-2 trigger rates for various single and combined L1 tau trigger items [6].

\subsection{Efficiency of the ATLAS Tau Trigger}

The tau trigger efficiency is the probability for a fully reconstructed and identified tau to pass the online trigger requirements, and is an important measure of the performance of the trigger system. Figure 2 shows the efficiency of the ATLAS tau trigger 'tau25_medium1_tracktwo' for the combined L1 and HLT stages measured in data and compared to simulation, with respect to fully reconstructed tau candidates with transverse 
momentum above $20 \mathrm{GeV}$, one or three charged tracks, and passing a 'medium' identification criteria at full event reconstruction. The corresponding tau candidate passing the 'tau25_medium1_tracktwo' trigger is required at trigger level to have a transverse momentum of at least $25 \mathrm{GeV}$, between one and three charged tracks, and to pass the online 'medium' identification. The trigger efficiency is measured in an enriched sample of $Z \rightarrow \tau \tau \rightarrow \mu v_{\mu} \nu_{\tau} \tau_{\text {had }} \nu_{\tau}$ events, where one tau decays leptonically and the other hadronically, recorded in some of the first $13 \mathrm{TeV}$ collisions in 2015. Monte Carlo simulated backgrounds are subtracted from this enriched data sample, and the efficiency, measured as a function of the transverse momentum of the reconstructed tau candidate, is compared to a Monte Carlo simulation of $Z \rightarrow \tau \tau$ decays. The methodology, which gives a purity of $75 \% \mathrm{Z} \rightarrow \tau \tau$ decays, is described in reference [5].

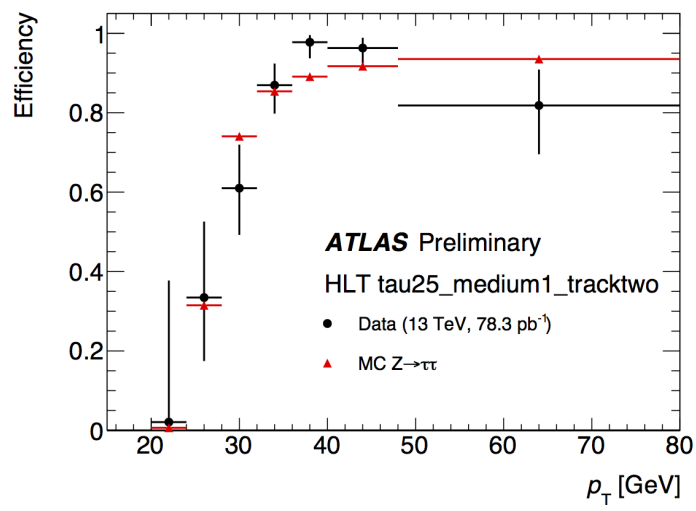

Figure 2: The tau trigger efficiency for the 'tau25_medium1_tracktwo' tau trigger measured in data and compared to simulation, in an enriched sample of $Z \rightarrow \tau \tau \rightarrow \mu v_{\mu} v_{\tau} \tau_{\text {had }} v_{\tau}$ events [6].

\subsection{Tau Trigger HLT Data and Simulation Comparison}

Figure 3 shows the transverse momentum and pseudo-rapidity distributions of online tau candidates passing the 'tau25_medium1_tracktwo' trigger in data and Monte Carlo simulation. The event selection utilises the "tag and probe" method whereby one tau lepton decays via a "tagged" muon passing the muon trigger, and the "probe" tau is fully reconstructed and accepted by the tau trigger. Figures $3 a$ ) and $3 b$ ) show online candidate tau leptons observed in an enriched sample of $\mathrm{W} \rightarrow \mu v_{\mu}+$ jets events. These candidate tau leptons are likely to be quark or gluon initiated jets misidentified at trigger level and form the dominant background in identifying tau jets. Figures 3c) and 3d) show online candidate tau leptons passing a 'medium' BDT identification observed in an enriched sample of $Z \rightarrow \tau \tau \rightarrow \mu \nu_{\mu} v_{\tau} \tau_{\text {had }} v_{\tau}$ events; these candidates likely originate from real tau decays. The methodology is described in reference [5].

\section{Summary}

Numerous changes to the tau trigger have occurred in preparation for the higher rates and luminosities of Run-2, including a variable L1 isolation requirement, a redesigned HLT selection, and an improved online BDT algorithm. Analysing the initial data collected by the ATLAS detector during Run-2, the ATLAS tau trigger is performing well. The online transverse momentum and pseudo-rapidity distributions of tau candidates are well modelled when extracted from $\mathrm{Z} \rightarrow \tau \tau$ kinematic control regions, as well as from "fake tau" $\mathrm{W}+$ jets kinematic control regions. The $\mathrm{L} 1$ topological trigger is still in the commissioning phase and will begin 


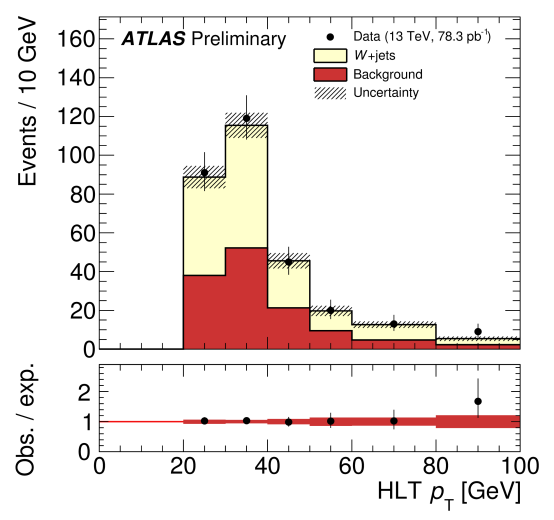

a)

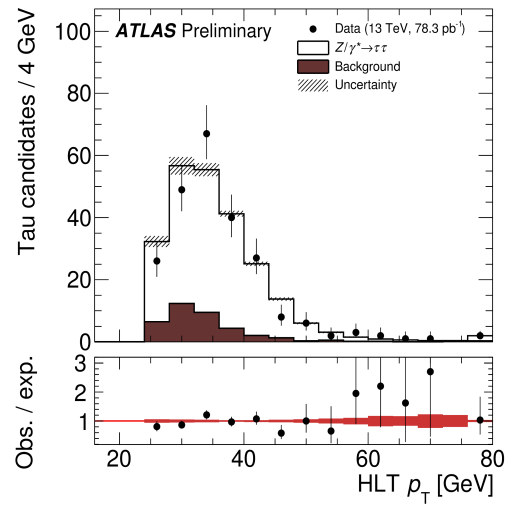

c)

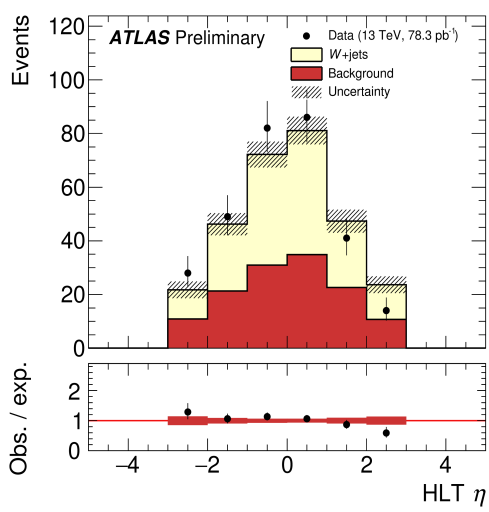

b)

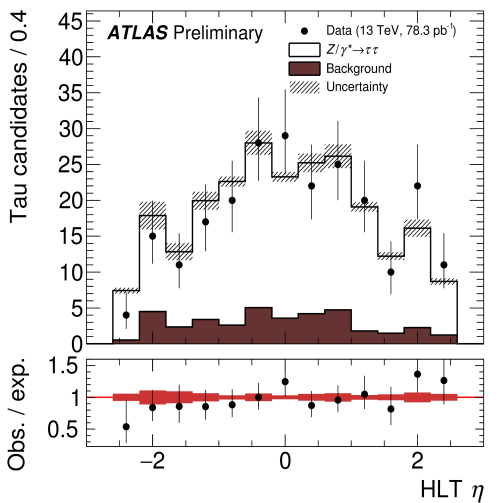

d)

Figure 3. Online distributions of the a) transverse momentum and b) pseudo-rapidity of "fake" tau leptons from $\mathrm{W} \rightarrow \mu \nu_{\mu}+$ jets events, and online distributions of c) transverse momentum and d) pseudo-rapidity of "real" tau leptons in $Z \rightarrow \tau \tau \rightarrow \mu v_{\mu} \nu_{\tau} \tau_{\text {had }} \nu_{\tau}$ events [6].

operation in 2016. Studies of the efficiency of the tau trigger are continuing as more data is collected during Run-2.

\section{References}

[1] ATLAS Collaboration, The ATLAS Experiment at the CERN Large Hadron Collider, JINST 3 (2008) S08003.

[2] ATLAS Collaboration, ATLAS High-Level Trigger algorithms for Run-2 data-taking, (2015) ATLDAQ-PROC-2015-018.

[3] ATLAS Collaboration, Technical Design Report for the Phase-I Upgrade of the ATLAS TDAQ System, (2013) CERN-LHCC-2013-018

[4] ATLAS Collaboration, Identification and energy calibration of hadronically decaying tau leptons with the ATLAS experiment in pp collisions at 8 TeV, Eur. Phys. J. C75 (2015) 303. arXiv:1412.7086

[5] ATLAS Collaboration, Commissioning of the reconstruction of hadronic tau lepton decays in ATLAS using pp collisions at $\sqrt{s}_{s}=13 \mathrm{TeV}$, (2015) ATL-PHYS-PUB-2015-025.

[6] ATLAS Collaboration, Tau Trigger Public Results, (2015) https://twiki.cern.ch/twiki/bin/view/AtlasPublic/TauTriggerPublicResults. 